\title{
ORALIDAD Y ESCRITURA EN LA RED: TESTIMONIOS DE (ORTO)GRAFÍA REGIOLECTAL EN UN CORPUS DE TWITTER
}

\author{
Elena Fernández de Molina Ortés \\ Universidad de Burgos
}

Recibido: 22/04/2015

Aceptado: 02/09/2015

\begin{abstract}
Resumen
El uso de las nuevas tecnologías y la presencia cada vez más común de las redes sociales en la actividad diaria de la población mundial ha producido una modificación de la interacción comunicativa del siglo XXI. Los usuarios, mediante la escritura, comparten sus estados de ánimo, sus experiencias y sus preocupaciones con los demás miembros de su red social; en este tipo de comunicación, como se ha podido comprobar desde la aparición de los servicios de mensajería instantánea así como en la escritura en foros virtuales o en blogs, se representan rasgos de oralidad en los que, creemos, deben incluirse las variedades regionales como testigos directos de estas tendencias. Por ello, en esta investigación se realizará un estudio de un corpus de mensajes de una red social concreta, Twitter, para comprobar si los rasgos lingüísticos de ciertas zonas geográficas de España aparecen en los mensajes de los usuarios de esta plataforma de comunicación virtual.
\end{abstract}

PALABRAS CLAVE: redes sociales, variación lingüística, Twitter, variación lingüística

\begin{abstract}
The use of new technologies and permanence of social media in the daily activities of the global population has been a change of communicative interaction of the $21^{\text {st }}$ century. Users, through writing, share their states of mind, their experiences and concerns with other members of their social media; in this type of communication (as has been proved since the advent of instant messaging and writing in online forums and blogs) are represented features of orality in which we include regional varieties because the are direct witnesses of these trends. Therefore, in this research we will make a study of a corpus of messages from a particular social media, Twitter, to check if the linguistic features of some geographical areas of Spain appear in the messages from users of this platform of virtual communication will take place.
\end{abstract}

KEY WORDS: social media, linguistic variation, Twitter, language variation

\section{Las redes sociales. Una nueva forma de comunicación a través de internet}

La llegada de las redes sociales ${ }^{1}$ a la sociedad 2.0. ha supuesto una modificación completa de las formas de interacción social del siglo XXI. Estas plataformas de comunicación

1 En la actualidad, las redes sociales más populares están compuestas por millones de usuarios en todo el mundo. Según los datos del V Estudio Anual de Redes Sociales de la IAB, en España, en el año 2014, las más usadas fueron Facebook (94\%), Youtube (68\%) y Twitter (41\%); otras como Linkedin, Tuenti, Instagram o Spotify, aun estando integradas por un menor número de usuarios, son igualmente populares. Actualmente, además, no se puede olvidar la expansión de la aplicación de mensajería instantánea Whatsapp, que cuenta ya con 400 millones de usuarios, una cifra similar a la de Twitter. 
agrupan a usuarios con intereses comunes, personales y profesionales, creando un espacio online donde comparten experiencias, contenidos e informaciones relevantes ${ }^{2}$ y en el que, además, el uso de la escritura virtual toma un papel fundamental.

La expansión de estas nuevas formas de comunicación virtual, favorecida durante los últimos años por el acceso a internet desde cualquier dispositivo electrónico (smartphone, tablet), ha producido, incluso, que las relaciones sociales se modifiquen y se cree un espacio paralelo de interacción, un espacio virtual.

Las comunidades en entornos físicos están sujetas a un proceso incesante de virtualización, es decir, han perdido definitivamente el anclaje físico que las limitaba y el peso que antaño poseían en la vida cotidiana de los ciudadanos (...) ahora más que nunca, las personas buscan redes sociales físicas en lugares ubicados geográficamente de forma dispersa. Además, utilizan masivamente tecnologías como el teléfono móvil, que suprime claramente el anclaje físico del receptor y subraya, en su lugar, la importancia del destinatario con independencia de su ubicación física. Entornos de interacción tan tradicionales como el bar, la plaza, la comunidad de vecinos, etc., han dejado de ser esenciales para la realización de las necesidades comunitarias o interactivas de los ciudadanos del siglo XXI (Yus, 2011: 51).

Con esta afirmación, Yus representa, en parte, el panorama de comunicación actual; como advierte el autor, cada vez es mayor la comunicación y socialización de grupos de amigos o familiares mediante la virtualización de sus relaciones. Sin embargo, aunque es cierto que internet y sus utilidades (redes sociales, páginas web, blogs, periódicos digitales) forman parte de la actividad diaria de la mayor parte de la población mundial, su extensión debe ser considerada como una forma de comunicación alternativa a otras formas de interacción social. Es cierto que cada vez es más común ver a grupos de jóvenes (y no tan jóvenes) sentados en una mesa sin hablar entre ellos pero sí a través del móvil pero, de igual manera, podemos continuar viendo a un grupo de amigos que se divierten en un bar o en un restaurante. Ello no quiere decir que, mientras tanto, no usen las redes sociales o el móvil sino que, aunque la comunicación virtual está presente, también lo está la presencial.

Además, y como se podrá comprobar a lo largo de esta investigación, es necesario tener en cuenta que toda comunicación virtual es una verbalización de la lengua oral; basándonos en este precepto y tras realizar una descripción de las principales características de la escritura online, presentaremos los resultados de la investigación sobre la presencia de la variación regiolectal en las redes sociales, más concretamente, en la plataforma de microblogging Twitter, para comprobar si uno de los testimonios de oralidad más auténticos, las variedades regionales, aparecen en la escritura de esta red social.

2 Castañeda y Gutiérrez (2010 citado en Cassany, 2011: 233), establece una distinción entre tres tipos de redes sociales según el enfoque de cada una de ellas. En las primeras, el autor destaca aquellas que se basan en la interacción virtual entre personas (Facebook, Tuenti); por otra parte, hace referencia a aquellas en las que existe un mayor interés por el intercambio de experiencias, noticias, sitios o actividades (Twitter) y, finalmente, existen otras plataformas dirigidas a compartir contenidos como textos, fotos o vídeos encabezadas, en estas casos, por redes como Youtube, Pinterest o Slideshare entre otras. A estas, y según Davis y Merchan (2009), habría que añadir las redes sociales de tipo profesional (Linkedin) y académico (Academia.edu). 


\section{La oralidad y la escritura en la red}

Independientemente del tipo de red social que utilice el usuario, la función y el objetivo que estas cumplen es siempre el mismo: comunicar. La escritura de las redes sociales, heredera -en parte- de la de los servicios de mensajería electrónicos (IRC, Messenger), es la vía de comunicación que millones de personas eligen para compartir sus mensajes con individuos conocidos y, en mucho casos, también desconocidos. No obstante, si bien es cierto que en estas plataformas es cada vez más común encontrar información mediante contenidos, el texto es un elemento esencial que introduce las imágenes, los vídeos o los enlaces de interés que comparten los usuarios con sus seguidores, permitiendo presentar dichos contenidos, describirlos, valorarlos e, incluso, criticarlos.

Pero en este tipo de textos, sin embargo, se rompe con la idea tradicional de que el discurso escrito se corresponde con un modo de expresión reflexivo y formal (López Quero, 2013: 80-81); en estas interacciones, que forman parte de los géneros electrónicos, se advierte una tendencia hacia la oralización de los mensajes que escriben los usuarios. Algunos autores han afirmado que los textos escritos en la red son "textos escritos oralizados" (Yus, 2001, 2011; Gómez Torrego, 2006); otros investigadores han acuñado los términos "conversación escrita" (Merchant, 2001, Blanco, 2002, López Quero, 2013) o “conversación tecleada" (Stein, 2006) para referirse a este tipo de comunicación pero, independientemente de la denominación ofrecida para representar esta realidad, en todos los casos se incide en la existencia de una naturaleza híbrida entre lo oral y escrito en los textos utilizados en la red, tal como afirma Yus en las siguientes líneas:

El aspecto más sobresaliente de esta variedad de comunicación electrónica [conversación virtual o chat] es la oralización del texto, esto es, la sensación de que los usuarios escriben lo que desearían estar diciendo, y leen lo que les gustaría estar oyendo (Yus, 2001).

Sin embargo, aunque es evidente la presencia de la lengua oral en los textos virtuales, existen diferentes tipos de escritura oralizada en la red. Esta realidad está determinada, entre otros factores, por el tipo de comunidad virtual en la que los individuos escriben sus mensajes $^{3}$. Yus (2001: 34-35), partiendo de la distinción de Cicognani (1998), hace referencia a dos tipos de comunidades basándose en el carácter más o menos efímero de las comunicaciones realizadas en cada una de ellas. Por una parte, en las comunidades sincrónicas (chats) se establecen diálogos interactivos que desaparecen en el momento en el que los usuarios abandonan la conversación por lo que, en este caso, no existe una huella de la presencia del escritor en la red; en cambio, en las comunidades asincrónicas (foros de debates, correos electrónicos), se registra un historial de nuestros textos que pueden ser cotejados, en momentos posteriores, por el propio escritor pero, también, por usuarios externos.

Esta distinción influye, según López Quero (2004: 66), en el uso de mensajes más o menos oralizados en la red. Por una parte, el carácter transitorio y el anonimato de los escritores de los chats o, como polo opuesto, la cercanía de los interlocutores que realizan sus interacciones mediante los servicios de mensajería instantánea (Whatsapp o SMS) implica que los usuarios de este tipo de plataformas tengan una menor presión lingüística $\mathrm{y}$, como

3 Tascón (2012: 157) indica que otros factores que influyen en la mayor o menor informalidad de los textos de los usuarios serían el tipo de red, su función y el tipo de usuario. 
consecuencia, haya una mayor presencia de muestras de oralidad. En cambio, otras plataformas como los correos electrónicos o los blogs, por ejemplo, donde el texto escrito sí se convierte en una seña de identidad del usuario en mensajes que, además, serán permanentes en la red, exigen una mayor reflexión lingüística por parte del escritor ${ }^{4}$.

En las redes sociales, en cambio, la interacción lingüística entre los interlocutores es diferente; en estas plataformas, el grado de oralidad que los emisores representan en sus mensajes se sitúa en un punto intermedio entre la conversación del chat y la escritura, por ejemplo, de un correo electrónico o de una entrada en un blog. Por una parte, hay que tener en cuenta que, en las redes sociales, los usuarios suelen tener una relación común que, en muchos casos, es cercana (relaciones de parentesco, amistad), lo cual contribuye a una mayor cercanía comunicativa entre los interlocutores y, por tanto, a un mayor índice de oralidad en sus comentarios. La oralidad representada en estos casos se basa en la transmisión de información, que viene marcada por la inmediatez comunicativa y la simultaneidad de emisión y recepción del mensaje (Bustos Tovar, 1995: 14).

En cambio, también es necesario advertir que las redes sociales son comunidades virtuales asincrónicas (Cigognani, 1998; Yus, 2001) en las que los mensajes creados por cada usuario permanecen en la red y marcan su identidad lingüística ante su grupo de amigos o seguidores ${ }^{5}$. Por ello, aunque en las redes sociales se pueden hallar textos escritos oralizados, igualmente existirá una preferencia por una escritura más reflexiva y cuidada que en otras plataformas de interacción comunicativa virtual ${ }^{6}$. A partir de estas consideraciones, a continuación destacaremos las principales características y funciones comunicativas de la red social Twitter para esbozar una primera aproximación sobre qué elementos lingüísticos podremos encontrar en los comentarios de sus usuarios centrándonos, más concretamente, en las características de la (orto)grafía regional y su representación en esta red social.

\subsection{La escritura en la red social Twitter}

Twitter es un servicio en línea de microblogging que permite enviar y recibir mensajes de 140 caracteres describiendo “¿qué está pasando?”. En esta red social, los usuarios pueden conocer las novedades que comparten amigos y familiares, personajes públicos e, incluso, noticias relevantes según los gustos e intereses del individuo (deportes, política, televisión

4 Gómez Torrego (2006) distingue entre la escritura en papel y en internet; el autor advierte que, frente a la actitud "litúrgica" y reflexiva de la escritura de una carta en papel, por ejemplo, donde importa no solo el contenido escrito sino también la estructura y las características estilísticas del texto, en un correo electrónico la escritura se realiza con una mayor precipitación, olvidando la estructura narrativa. En estos casos, tanto al escritor como al receptor no les interesa la forma en la que está expresado y estructurado el mensaje sino, únicamente, el contenido.

5 En las redes sociales, al igual que ocurre en las conversaciones en tiempo real a través de chats, muchos usuarios olvidan el testimonio lingüístico que ofrecen en estas plataformas de comunicación y revelan la falta de concentración o la dejadez de quien escribe desde la proximidad comunicativa (Mancera, Pano, 2014: 310).

6 Además, el mayor o menor grado de oralización de los textos escritos en las redes sociales estarán determinados, también, por el tipo de red social en la que se produce la comunicación (profesional o personal), la función que cumple (compartir contenidos o experiencias -Facebook-) así como el tipo de usuario que está integrado en dicha comunidad. Un ejemplo de ello puede ser la red social Tuenti, compuesta fundamentalmente por adolescentes y universitarios en cuyos comentarios podemos advertir una mayor tendencia a la oralización textual; así se puede advertir en la presencia de heterografias o cambios ortográficos voluntarios que utilizan los usuarios de esta red social para realizar representaciones orales en sus comentarios (Fernández de Molina, 2015). 
etc.). Además, en sus mensajes describen ideas y experiencias pero, también, comparten realidades que han escrito otros usuarios haciendo un retuit de un mensaje externo o marcándolo como favorito. Por otra parte, es necesario advertir que Twitter se ha convertido, igualmente, en una plataforma de información sobre la actualidad social; mediante los llamados Trending Topic, los usuarios pueden conocer las tendencias y los temas más importantes del país pero, también, pueden encontrar comentarios mundiales sobre un asunto preferido a partir de los hashtags o etiquetas creados por otros usuarios de la red (mediante la escritura del símbolo \#) ${ }^{7}$.

Como afirma Tíscar Lara (2012), Twitter es una plataforma que, aunque en un primer momento representa un espacio privado, al interactuar en la red escribiendo mensajes o, simplemente, retuiteando los contenidos de otras personas, la información personal del usuario se convierte en pública. La autora identifica varias funciones comunicativas de esta red social como la del reconocimiento, que se produce al retuitear mensajes de otros reconociendo su autoridad y atribuyendo, además, una responsabilidad sobre lo compartido; incide la autora, de igual forma, en la función dialógica y la apelativa de Twitter, representada mediante el uso de la estructura “@nombre” que sirve, bien como referencia a un usuario o, simplemente, para conversar entre una o más personas y, finalmente, destaca que el uso de etiquetas mediante la unión de la almohadilla y una palabra clave (\#palabraclave) permite a los usuarios relacionar conversaciones e ideas de un tema común con otros individuos en la red social e, incluso, "entrar espontáneamente en conversaciones ajenas con el simple uso de su misma palabra clave" (Lara, 2012).

En este servicio, por tanto, los usuarios producen pero también reciclan información para comunicar (a partir de los retuits), es decir, no son receptores de información sino que se convierten en productores, comentadores y consumidores de la misma (Galindo, 2014; Mancera y Pano, 2013). No obstante, hay que tener en cuenta que esta red social es, como se indicó en secciones anteriores, una comunidad virtual asincrónica en la que los usuarios tienen muy en cuenta cómo y qué escriben en su perfil aunque es cierto que se advierte una tendencia general hacia el uso del registro coloquial en las interacciones entre usuarios ${ }^{9}$ rompiendo, según la perspectiva de Koch y Oesterreicher (1985), con la dicotomía entre lo oral y escrito en la red y representando un continuum lingüístico de uso entre la distancia y la inmediatez comunicativa. Según la clasificación de los autores, existen grados que influyen en la mayor o menor presencia de oralidad en los textos: la privacidad o el carácter público del discurso, el nivel de emotividad y participación de los interlocutores, la posición local o situacional de los hablantes, la cooperación, la espontaneidad o la determinación del tema tratado en la conversación. Teniendo en cuenta que estos factores son graduales, Briz (2010) afirma que lo coloquial estaría directamente relacionado con la inmediatez comunicativa, en la que, entre otras características, los temas tratados en la conversación son presentados

7 El principal motivo para usar Twitter, según Mancera y Pano (2013:14) es “informarse y promover o apoyar causas sociales y solidarias además de seguir a personajes famosos" a diferencia de Facebook o Tuenti, que se emplean para reforzar el contacto con el círculo social cercano, actualizando el perfil y compartiendo fotografías".

8 Hay que tener en cuenta, además, que Twitter es una red social considerada simétrica, es decir, no existen diferencias jerárquicas entre los usuarios, que pueden interactuar con cualquier individuo de la red que tenga su perfil abierto (Galindo, 2004).

9 Se debe entender el registro coloquial desde un punto de vista diafásico del uso de la lengua, esto es, teniendo en cuenta el contexto comunicativo en el que el hablante actúa y rechazando la consideración de este tipo de registro o nivel de habla como formas vulgares o populares. 
espontáneamente, la comunicación se planea sobre la marcha (a diferencia, por ejemplo, de un discurso público) o el fin de la conversación y el tono utilizado son menos formales.

Según esta perspectiva, en Twitter se podrá observar ese continuum entre lo oral y escrito en diversas situaciones; así, teniendo en cuenta que los mensajes publicados suelen estar dirigidos a usuarios conocidos pero, también, desconocidos (que pueden encontrar los tuits, por ejemplo, mediante etiquetas), en los mensajes de la red social se podrán observar interacciones menos coloquiales en el segundo de los casos pero, también, más coloquiales, sobre todo en las conversaciones realizadas entre individuos conocidos que mantienen gustos e intereses comunes.

\subsection{La (orto)grafía regiolectal y su presencia en Twitter}

Teniendo en cuenta la tendencia que existe en las redes sociales por representar de forma escrita mensajes orales, Yus (2012), a partir de la clasificación de Androutsopoulos (2000: 521-522), identifica varios tipos de rasgos (orto)gráficos que se pueden encontrar en los géneros electrónicos: (orto)grafía fonética, regiolectal, prosódica, interlingüística e incluye, además, el uso de abreviaciones, elipsis e, incluso, faltas de ortografía.

Aunque si bien es cierto que en la red social Twitter se pueden encontrar ejemplos de fenómenos estudiados, sobre todo, en los análisis sobre las conversaciones virtuales como el uso de la grafía $k$ para representar la qu (ke pasa), la $x$ para el dígrafo ch (dixo), la ausencia de vocal ( $k$ 'que'), la $w$ para el sonido velar /g/ (iwal), la omisión de la $h$ (aora) y algunas vocales ( $b n$ 'bien', tekero 'te quiero') que suelen atribuirse a la economía lingüística (Mancera, Pano, 2013 $)^{10}$ y que, según Martínez de Sousa (2004), se tratarían de heterografías, es decir, errores ortográficos que no se cometen por el desconocimiento de la norma sino por la discrepancia de uso con las reglas ortográficas en el momento de la escritura, en nuestra investigación nos centraremos, concretamente, en el uso de la (orto)grafía regiolectal, es decir, en las representaciones gráficas de las variaciones regionales de una lengua (Yus, 2011: 199) porque, si bien es cierto que la escritura en las comunidades virtuales está directamente relacionada con la oralidad, creemos que uno de los rasgos que constituyen la auténtica oralidad es la representación de variantes regionales o locales de una comunidad.

Además, en este tipo de (orto)grafía -también denominada dialecto visual (eye dialect) (Yus, 2011: 199)-, aparecen integrados otros tipos de (orto)grafías como la fonética, cuando los usuarios representan el discurso tal y como sería pronunciado de forma oral (¿qué hase illo?), la coloquial, en la que los escritores usan recursos como las reducciones de palabras, tal como ocurriría en la lengua oral (esto es to lo que te tengo que decir) o la prosódica, mediante la repetición de letras para reproducir, por ejemplo, enunciados interrogativos (pero, ¿qué pasaaaaa?) o exclamativos (achooooo, iqué fuerte!).

Aunque es cierto que, como hemos visto, en Twitter los usuarios poseen una mayor conciencia de su identidad lingüística, también creemos que, al igual que en otras redes sociales, en esta plataforma los mensajes oralizados seguirán estando presentes en la comunicación de los usuarios y, entre ellos, aparecerán muestras lingüísticas de sus variedades regionales.

10 En el ejemplo de Twitter, esta tendencia a la economía lingüística viene justificada por la limitación de caracteres a la hora de escribir los mensajes en la red social. 


\section{Metodología}

Para obtener resultados que garantizasen la evidencia de la oralización de la escritura en la red social Twitter a partir de los usos regiolectales que utilizan los usuarios en sus comentarios, primeramente se obtuvo un corpus de tuits de esta red social en un área geográfica determinada a partir del cual, posteriormente, se pudieron realizar los análisis pertinentes. A continuación se detallarán las fases de recopilación de datos.

\subsection{Selección del área geográfica de estudio}

En un primer momento, y teniendo en cuenta que el objetivo fundamental de la investigación era recopilar mensajes de los usuarios de Twitter que utilizaban variedades regionales, se establecieron las zonas geográficas que iban a ser objeto de estudio. En esta investigación se seleccionaron dos variedades regionales concretas, el extremeño y el andaluz, presentes en las comunidades autónomas de Extremadura y Andalucía respectivamente. Para realizar la distribución que nos permitiera recopilar los tuits de ambas regiones, se establecieron puntos geográficos concretos:

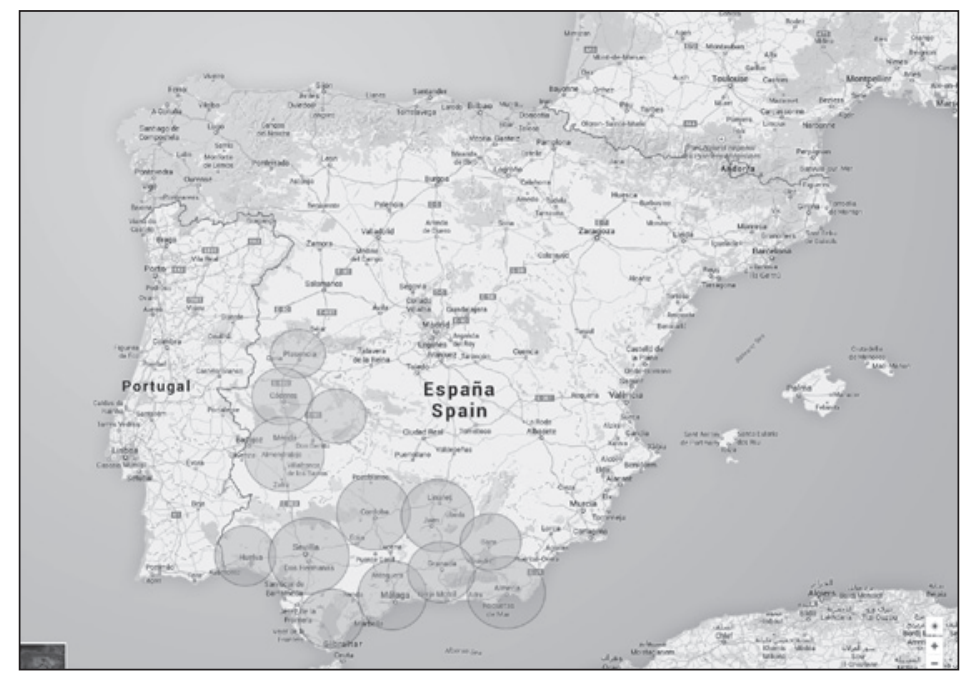

Imagen 1. Distribución geográfica de geolocalización para la recopilación de tuits.

Como se puede advertir en la imagen 1, en Extremadura se seleccionaron 4 centros geográficos y se trazó un radio de entre 30 y 60 km que incluyó las áreas de Plasencia (40 $\mathrm{km})$, Cáceres $(60 \mathrm{~km})$, Almendralejo $(30 \mathrm{~km})$ y Valdecaballeros $(30 \mathrm{~km})$. En Andalucía, en cambio, y debido a la mayor extensión de la comunidad autónoma, se seleccionaron 9 centros: Huelva $(50 \mathrm{~km})$, Sevilla $(70 \mathrm{~km})$, Algeciras $(50 \mathrm{~km})$, Málaga $(40 \mathrm{~km})$, Córdoba (40 $\mathrm{km})$, Granada $(60 \mathrm{~km})$, Almería $(60 \mathrm{~km})$, Jaén $(50 \mathrm{~km})$ y Baeza $(50 \mathrm{~km})^{11}$.

11 Como se puede comprobar, en Extremadura no se seleccionaron las capitales de provincia (únicamente Cáceres) sino que se utilizaron puntos geográficos que permitieran estudiar, en su conjunto, toda la región, independientemente del centro geográfico utilizado. 


\subsection{Recopilación del corpus}

Una vez distribuidos los puntos geográficos que iban a ser objeto de estudio, se utilizó la API Streaming de Twitter (Versión 1.1.) para la recopilación de los tuits. De esta forma, añadiendo las coordenadas de cada área estudiada (que fueron obtenidas a partir de Google Earth), pudimos completar la primera parte de recogida de datos ${ }^{12}$.

Además, y para cumplir con el objetivo de la investigación, se seleccionaron 10 palabras clave que nos permitieran extraer comentarios realizados por los usuarios intentando, en todo caso, que en ellos aparecieran variantes regionales. Este tipo de etiquetas representaban aspectos relativos a la vida diaria de los usuarios de la red social como actividades de ocio (fútbol, cerveza, viaje, casa), actividades profesionales y del ámbito educativo (trabajo, deberes, examen) o cuestiones atmosféricas (frío), que siempre influyen en el estado de ánimo de los individuos y, por tanto, en sus mensajes en la red. Además, se incluyó la expresión jajaja, que nos permitía encontrar mensajes irónicos y despreocupados y en los que podrían aparecer, al igual que en los demás temas, registros del habla oral y, por extensión, casos de variación regional.

Por otra parte, es necesario advertir que, para la recopilación de tuits, los usuarios de la red social debían tener su perfil desbloqueado (para poder acceder a sus mensajes) y, además, debían haber activado la opción de geolocalización de su cuenta de Twitter para obtener los datos por zonas geográficas; este último requisito fue uno de los más problemáticos porque muchos individuos no seleccionan esta opción al crear su cuenta en la red social, bien por causas personales (no querer ser localizados durante la publicación del tuit) o bien por motivos externos, como el consumo de batería de los aparatos electrónicos utilizados.

Finalmente, para analizar los resultados del corpus obtenido, utilizamos el parametrizador de textos en línea PARAMTEXT TIP (Carreras, Santana, 2011), una herramienta que permite extraer los datos lingüísticos mediante diversos formatos (.docx, .xlsx, .pdf etc.); este recurso ofrece informes detallados de los resultados (número de oraciones, palabras, palabras diferentes etc.) pero, además, permite obtener una lista con todas las voces obtenidas y su frecuencia de aparición que nos permitía, en nuestro caso, poder extraer y organizar (manualmente) las variantes del corpus utilizado.

\section{La conciencia lingüística de los usuarios de las redes sociales}

Antes de comenzar el análisis de datos y el procesamiento de los resultados obtenidos de Twitter, creímos conveniente conocer qué conciencia lingüística tenían los usuarios de redes sociales sobre el uso de la escritura en internet, es decir, queríamos comprobar cómo creían que era su escritura en estas plataformas de comunicación. Pero, además, consideramos que la conciencia lingüística (positiva o negativa) que tiene el hablante de su variación lingüística regional puede influir en su uso de la red social.

Por una parte, en cuanto a la consideración de la conciencia lingüística del hablante, como se ha podido comprobar en la sección metodológica de este estudio, para obtener los datos lingüísticos se utilizará un corpus de Twitter compuesto por los mensajes de usuarios de Andalucía y Extremadura, esto es, por individuos cuya variación lingüística se incluye dentro de las hablas meridionales. En estos casos se puede afirmar que, por una parte, el

12 Los tuits recopilados para esta investigación fueron recogidos durante el mes de febrero del año 2015. El procesamiento de datos fue realizado por la empresa informática JAEPREL S.L. 
hablante andaluz suele conservar los rasgos lingüísticos de variedad porque su conciencia lingüística suele ser grande, es decir, se siente orgulloso de su forma de hablar ${ }^{13}$; en cambio, el extremeño no tiene buena conciencia de su variedad regional por lo que intenta ocultar o corregir los rasgos lingüísticos que considera incorrectos aunque realmente formen parte de su variación lingüística (Ariza, 1987: 21-22). Si partimos de la concepción de que el habla en las redes sociales es un continuum entre lo oral y lo escrito y que en algunas áreas los individuos intentan omitir sus características lingüísticas, será más probable que los andaluces no reparen en ocultar sus formas lingüísticas en Twitter frente a los integrantes de Extremadura que, por una parte, intentarán excluir estos rasgos en su escritura pero que, además, no reconocerán usarlas en la red social.

Para conocer si, desde el punto de vista del propio usuario, estos emplean formas orales propias de su variación lingüística en las redes sociales, se creó un cuestionario con la herramienta online Typeform en el que se propusieron 22 preguntas divididas en cuatro áreas temáticas: información personal de los individuos (edad, sexo, comunidad autónoma de procedencia... $)^{14}$, el uso de internet (con qué frecuencia se conectaba el usuario a la red, para qué...), la conciencia lingüística de los usuarios en las redes sociales (qué tipo de escritura usa -oral o escrita-, cómo escribe el propio usuario y sus seguidores, la aparición de rasgos lingüísticos regionales etc.) y, por último, el uso concreto de las variaciones lingüísticas regionales en estas plataformas de comunicación.

El cuestionario fue completado por 103 individuos de las regiones de Castilla la Mancha, Castilla y León, Murcia, Extremadura y Andalucía aunque, no obstante, la mayor parte de individuos pertenecían a las dos últimas. Para la difusión del cuestionario se utilizó, primero, un evento creado en Facebook mediante el cual se invitó a amigos y conocidos de la investigadora de este estudio, que realizaron la encuesta pero que, también, la difundieron entre sus contactos. Además, y para obtener más información, durante 15 días se publicó el enlace web de la encuesta en Twitter, incluyendo en los comentarios etiquetas con los Trending Topic del día para que pudieran realizar la encuesta personas anónimas de esta red social.

Las encuestas fueron realizadas por hombres (36\%) y mujeres $(64 \%)$ de edades comprendidas, fundamentalmente, entre los 21 y 29 años (48,03\%); no obstante, también respondieron el cuestionario grupos de jóvenes menores de 19 años $(12,74 \%)$, individuos de entre 30 y 49 años $(21,56 \%)$ y usuarios mayores de 50 años (17,64\%). El 71\% había realizado estudios universitarios y el $25 \%$ educación secundaria y ciclos formativos.

Según las respuestas obtenidas, se pudo comprobar que la mayor parte de los encuestados $(83 \%)$ usan internet varias veces al día, fundamentalmente para la comunicación con familiares y amigos (85\%), por motivos laborales (48\%) y para sus estudios $(50 \%) \mathrm{u}$ otras actividades de ocio como realizar compras, ver películas, buscar información e, incluso, para jugar en línea. Además, según los datos recopilados, el 100\% de los informantes afirmaron ser miembros de las redes sociales, entre las que destacan Facebook (93\%), Twitter (60\%) e Instagram (31\%).

13 Hay fenómenos como el ceceo que, como veremos en los siguientes apartados, no gozan de tanto prestigio por lo que, en muchas ocasiones, los hablantes tienen una conciencia lingüística negativa de su variedad lingüística e intentan ocultar estas realizaciones.

14 Las encuestas eran totalmente anónimas; no obstante, datos como la edad o la procedencia geográfica de los informantes podían ser de gran utilidad para establecer comparaciones con las respuestas obtenidas sobre el uso de las redes sociales y la conciencia lingüística de los usuarios. 
Para conocer la conciencia lingüística de los usuarios, se plantearon algunas preguntas, tal como se puede observar a continuación:

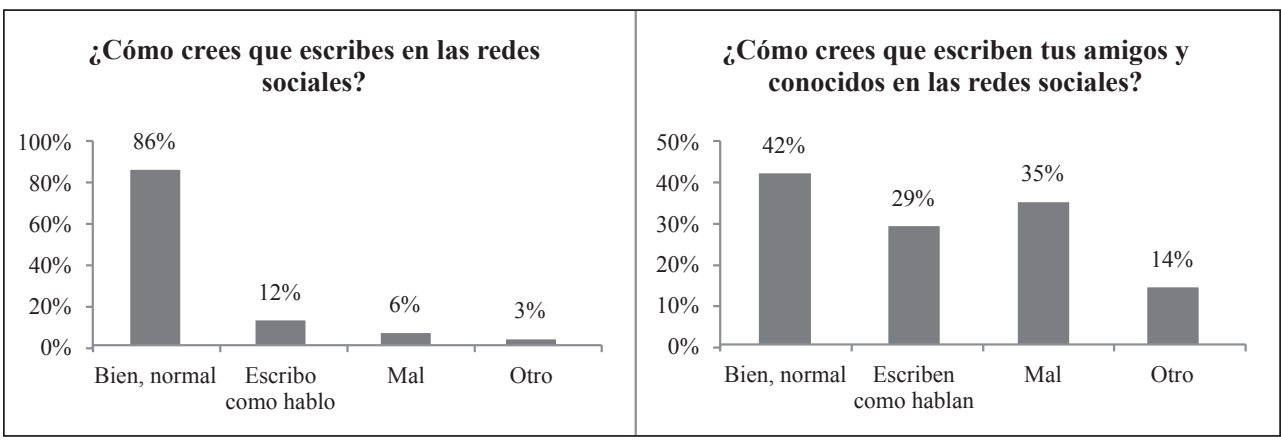

Gráficos 1 y 2. Resultados sobre la conciencia lingüística en las redes sociales.

Aspectos generales.

Según los resultados de los gráficos 1 y 2, existen diferencias evidentes entre la percepción que tienen los usuarios de cómo escriben en la red y de cómo escriben sus seguidores. Por una parte, los individuos suelen tener una conciencia lingüística positiva de su escritura (el 86\% consideran que escriben "bien") pero, en cambio, es realmente notable que únicamente el $42 \%$ piense que sus amigos escriben "bien" frente a un $35 \%$ que consideran que lo hacen "mal". Igualmente significativas son las respuestas sobre la oralización de la escritura y el uso que hacen los individuos de este tipo de lenguaje en internet.

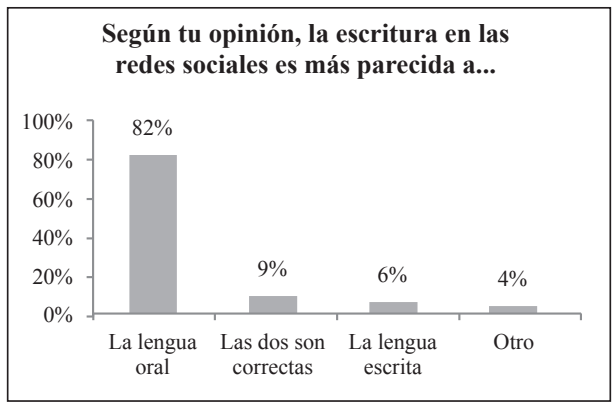

Gráfico 3. La escritura oral en las redes sociales. Opiniones de los encuestados.

En el gráfico 3 se puede comprobar que la mayor parte de los individuos declaran que la escritura en las redes sociales representa la lengua oral (82\%) y no la lengua escrita (6\%). Estas contestaciones difieren, no obstante, de las obtenidas en los gráficos 1 y 2, donde únicamente el $12 \%$ de los encuestados afirmaban "escribir como hablan" en estas plataformas de comunicación. Esta diferencia puede estar determinada por el hecho de que los usuarios, aunque aseguran que la escritura en las redes sociales tiene una relación directa con la lengua oral, no reconocen que emplean este tipo de variación en sus comentarios (aunque sí advierten este tipo de escritura en sus seguidores, tal como se ha podido comprobar en el gráfico 2) e intentan encubrir el uso lingüístico que hacen, verdaderamente, en estas plataformas de comunicación. 
Teniendo en cuenta estas consideraciones, se procedió a preguntar a los usuarios sobre el tema del trabajo de investigación: “¿utilizas variantes regionales (andaluz, extremeño, murciano, otros) en las redes sociales?".

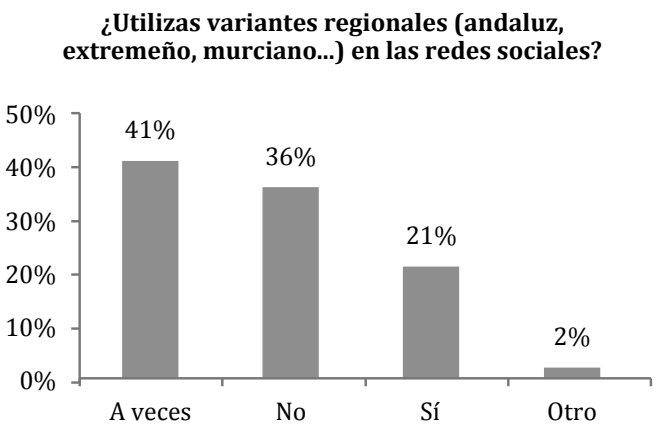

Gráfico 4. Opiniones sobre el uso de variantes regionales en las redes sociales.

Las contestaciones obtenidas, en este caso, se relacionan con las recopiladas en el gráfico 3. Ante la pregunta sobre el uso de formas regionales que, en un primer momento, debería haber producido un posible encubrimiento lingüístico por parte de los informantes pues el uso de este tipo de variantes es una representación de la lengua oral en la escritura, los encuestados afirmaron utilizarlas en un $62 \%$ de los casos; más concretamente, el $41 \%$ de los individuos respondieron que las utilizaban "a veces" y un $21 \%$ utilizaron la respuesta afirmativa.

Además, y según los resultados de la siguiente pregunta: "si has respondido "sí" o "a veces"... ¿con qué grupos las usas?", los informantes precisaron que, sobre todo, este tipo de variantes eran comunes en conversaciones con amigos (91\%) y familiares (46\%) e, incluso, en las realizadas con compañeros de trabajo o de clase (24\%). En este caso, por tanto, el uso de la lengua oral, proyectada concretamente mediante la selección de variantes regionales, aparece sobre todo en las interacciones de grupos integrados por personas conocidas y cercanas a los usuarios de la red social pero, en ningún caso, entre personas desconocidas. Este ejemplo constata la importancia de la identidad lingüística que se forja en estas plataformas de comunicación a través de la escritura de sus usuarios.

Finalmente, y para comprobar qué tipo de variantes regionales podían ser más o menos habituales en las redes sociales, se presentaron tres propuestas:

(1) Imagina que estás escribiendo en el muro o en una foto de un amigo o un familiar cercano en Facebook o Twitter. ¿Con qué comentario te sientes más identificado?

a. Acho, mañana vemos el partido no?

b. Illo, no sé si podré ir mañana pero cuando lo sepa te escribo

c. Ninguna de las dos son correctas

d. Otro

(2) Y entre las siguientes, ¿cuál utilizarías?

a. Hemos quedado sobre las tres y cuarto, ¿vienes?

b. Hemos quedao sobre las tres y cuarto, ¿vienes?

c. Otro 
(3) Selecciona la opción que usarías en una red social
a. Está la cosa muy mala
b. Está la cosa $m u$ mala
c. Ninguna de las dos son correctas
d. Otro

En cada pregunta se planteaba el uso de algunas de las variantes regionales más comunes sobre la escritura oralizada en internet; entre ellas quisimos destacar el uso de formas apelativas como illo para el andaluz y acho para el extremeño, el uso de la (orto)grafía fonética, representada por la pérdida de la -/d/- en posición intervocálica en la terminación -ado que, aunque es habitual en ambas regiones, está igualmente extendida por gran parte del español peninsular y, finalmente, la (orto)grafía coloquial, utilizada también en ambas regiones, con el uso de la reducción del adverbio muy.
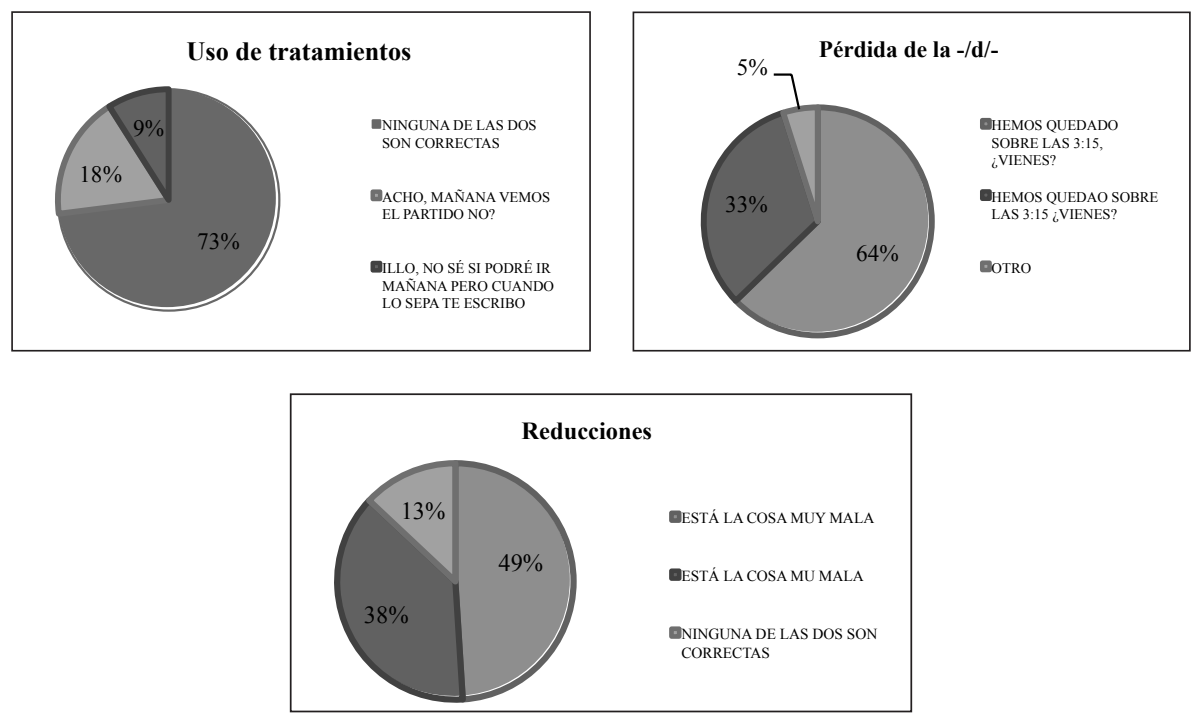

Gráficos 5, 6 y 7. Opiniones sobre el uso de (orto)grafía regiolectal en las redes sociales.

Según los resultados obtenidos de las opiniones de los encuestados, en las redes sociales no usan habitualmente tratamientos apelativos como acho, illo (gráfico 5) pero, en cambio, los usuarios sí confirman la presencia de la pérdida de la -/d/- en posición intervocálica (33\%) (gráfico 6) y las reducciones (38\% del 49\% de respuestas correctas) (gráfico 7). Por ello, y según estas consideraciones, en nuestro corpus de comentarios de Twitter será más habitual encontrar variaciones de tipo fonético -(orto)grafía fonética- que las referencias directas de rasgos regionales como representan los apelativos acho, illo y sus variaciones (killo, chacho...). Además, los informantes corroboraron las advertencias realizadas anteriormente: estas variantes son usadas, fundamentalmente, con familiares y amigos $(88 \%)$. 
Finalmente, para concluir las encuestas se realizó una pregunta fundamental para conocer si existían diferencias entre los tipos de interacciones lingüísticas que se producen en algunas redes sociales. Nuestro objetivo era comprobar si los usuarios utilizaban distintas formas de comunicación en dos de las redes sociales más populares en España, Facebook y Twitter que, como se ha podido comprobar en secciones anteriores, presentan ciertas diferencias tanto en sus objetivos como en los contenidos que en ellas se reproducen.

\section{¿Con qué frecuencia usas variantes regionales en Facebook y Twitter?}

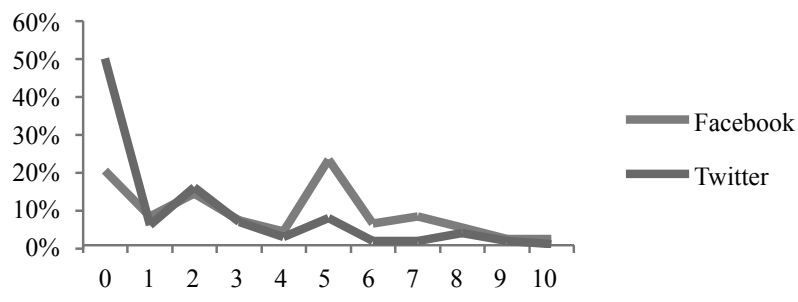

Gráfico 8. Frecuencia de uso de variantes regionales en Facebook y Twitter.

Según los datos que se presentan en el gráfico 8, la mayor parte de los encuestados afirmaron que, en la red social Twitter, "nunca" utilizarían variantes regionales; no obstante, si se observan los datos sobre Facebook, únicamente un $20 \%$ de los indiviudos no usarían "nunca" este tipo de formas en la red social y un $23 \%$ las usarían "a veces".

De nuevo, estos resultados avalan las hipótesis anteriormente planteadas: aunque Twitter es una plataforma de comunicación virtual, el carácter asincrónico de la red social, la relación de los usuarios con sus seguidores (que no son siempre amigos y familiares como parece ocurrir en Facebook sino que, en muchos casos, son personas desconocidas) y, además, la identidad lingüística que representa el individuo en sus interacciones en la red social, que se crea a través de los mensajes que escribe para relacionarse con sus seguidores, implica el uso de una escritura oralizada más reflexiva que en otros servicios de comunicación virtual.

A continuación comprobaremos si estas afirmaciones se corresponden con los resultados obtenidos del corpus analizado para esta investigaicón.

\section{Análisis y resultados}

Tras la parametrización automática de los mensajes obtenidos de Twitter, el análisis del corpus se realizó manualmente; a través de los datos que nos ofrecía la herramienta PARAMTEXT TIP se interpretaron los casos que habían aparecido en los resultados centrándonos, evidentemente, en las variantes regionales utilizadas en los tuits recopilados. A continuación, tras la descripción general de los rasgos más significativos encontrados en los mensajes de Twitter, se presentarán los resultados sobre el uso de la (orto)grafía regiolectal a partir de la representación (orto)gráfica de rasgos fonéticos, morfológicos y léxicos; en este último apartado, además, se ofrecerán casos de expresiones que pueden ser consideradas, igualmente, regionales, para completar así los resultados de la investigación. 


\subsection{Resultados totales}

El corpus utilizado para el estudio de las variedades regionales en las redes sociales estuvo compuesto por un total de 14299 tuits; a continuación se puede comprobar el índice de frecuencia de aparición de las palabras clave utilizadas para obtener los resultados:

\begin{tabular}{|cc|}
\hline \multicolumn{2}{|c|}{ CORPUS TOTAL: } \\
14299 TWEETS \\
\hline jajaja & 3383 \\
\hline casa & 3293 \\
\hline fútbol & 2489 \\
\hline frío & 1251 \\
\hline examen & 1197 \\
\hline trabajo & 1115 \\
\hline viaje & 561 \\
\hline compras & 473 \\
\hline cerveza & 348 \\
\hline deberes & 210 \\
\hline
\end{tabular}

Tabla 1. Palabras clave y frecuencia de aparición.

Como se puede observar en la tabla 1, las palabras clave que nos permitieron recopilar más tuits fueron "jajaja", que compone el 23,62\% del corpus, "casa" (22,99\%), y "fútbol" (17,38\%); este tipo de variables se refieren a actividades sociales y de ocio, temas que predominan en los mensajes que los usuarios suelen utilizar para expresar sus actuaciones diarias en la red social ${ }^{15}$.

Por otra parte, en cuanto al uso de formas regionales en el corpus recopilado, se obtuvo un total de 1402 variantes, que corresponden a un 9,79\% de los datos recopilados.

\section{Resultados del análisis fonético, morfológico y léxico}

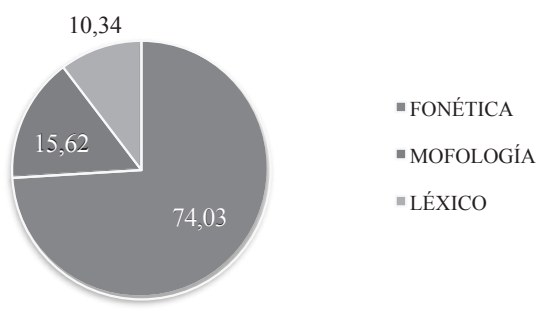

Gráfico 9. Resultados totales de las representaciones fonéticas, morfológicas y léxicas.

15 Es necesario advertir que, según el área estudiada, la mayor parte del corpus estuvo compuesto por mensajes procedentes de la comunidad autónoma de Andalucía, donde se recogieron un total de 12044 mensajes frente a los 2255 obtenidos de Extremadura. Esta diferencia proviene, evidentemente, de la mayor extensión geográfica de Andalucía. 
Entre las representaciones (orto)gráficas, tal como se puede comprobar en el Gráfico 9, predominan fundamentalmente los usos fonéticos $(74,03 \%)$ frente a los morfológicos $(15,62 \%)$ y los léxicos $(10,34 \%)$, que tienen un menor índice de aparición. Según estos resultados, se podría considerar, en un primer momento, que los usuarios que utilizaron este tipo de variantes sí emplearon, por tanto, formas de oralidad en sus mensajes escritos en la red social.

\subsection{Análisis y resultados lingüísticos}

A continuación se presentarán los resultados obtenidos sobre los usos fonéticos, morfológicos y léxicos en los mensajes de Twitter. Según los datos presentados en el apartado anterior, nos centraremos fundamentalmente en las variantes fonéticas, predominantes en el corpus, aunque, igualmente, analizaremos ciertos aspectos sobre las apariciones de formas morfológicas y léxicas.

\subsection{1. (Orto)grafia regiolectal. Rasgos fonéticos}

Tras el análisis del corpus realizado, se han obtenido las siguientes variantes fonéticas:

\section{Resultados totales fonética}

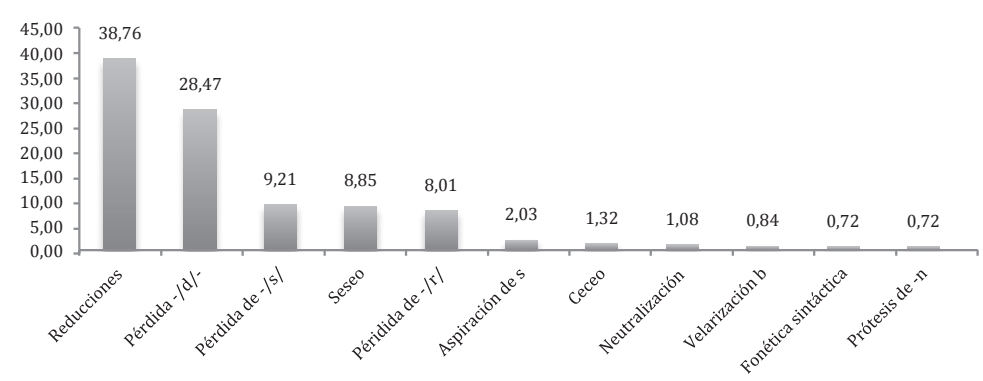

Gráfico 10. Resultados totales de rasgos fonéticos.

Como se puede comprobar en el gráfico 10, la mayor parte de los rasgos fonéticos recopilados pertenecen, sobre todo, al registro coloquial; entre ellos destacan, fundamentalmente, las reducciones de preposiciones y adverbios como para, todo o muy, que componen el $38,76 \%$ de los datos y la pérdida de la -/d/- en posición intervocálica $(28,47 \%)$ que, aunque es un fenómeno extendido por el español peninsular, tiene una mayor presencia en las hablas meridionales. Existen igualmente resultados de pérdida de consonantes finales como -/s/ (9,21\%) o -/r/ (8,01\%), de la aspiración de la -/s/ en posición implosiva (que los usuarios representaron con las grafías $h \mathrm{y} j$ ) y, también, casos de neutralización de l-r (arma), velarizaciones de la oclusiva /b/ en posición inicial (guenos días gente) o la prótesis de $-n$ en posición final, sobre todo en el adverbio "así" (asín). No obstante, estos últimos rasgos no son tan frecuentes y, además, aunque su uso ha sido generalmente asignado a las hablas meridionales, son formas características, preferentemente, del español vulgar. 
Finalmente, es necesario hacer referencia a la presencia del seseo y el ceceo en el corpus; como se puede observar en el gráfico, la representación del seseo $(8,85 \%)$ es mucho más frecuente que el ceceo (1,32\%). Estas formas fueron localizadas en Andalucía (área propia de este tipo de variación) pero, según los puntos geográficos que seleccionamos, no se ha podido observar una distribución lingüística homogénea de los fenómenos. Esta ausencia de localización uniforme de ambos rasgos puede reflejar que los usuarios pudieron escribir este tipo de variaciones sin atender a su variedad lingüística propia, es decir, presentaron el seseo y el ceceo independientemente del uso real que hacen de ambos fenómenos. Sin embargo, es necesario advertir que la mayor frecuencia del seseo frente al ceceo puede estar producida, seguramente, por el encubrimiento lingüístico de algunos individuos de la red social que existe, igualmente, en la lengua oral; esto ocurre porque, aunque estos rasgos representan lingüísticamente la zona meridional de la Península, es cierto que el seseo goza de mayor prestigio que el ceceo, lo que provoca, en algunos casos, el uso de ultracorrecciones e incluso de fenómenos como el ceseo o el seceo ${ }^{16}$. Por esta razón, se podría aceptar que usuarios que oralmente cecean, usaron el seseo para representar un fenómeno más prestigioso ligado a su variedad regional pero no a su variación lingüística individual.

Para observar la extensión de ciertos fenómenos, a continuación presentaremos los resultados de los rasgos que gozan de una mayor presencia en el corpus.

\section{a. Reducciones fonéticas}

En los análisis de los tuits utilizados como base para este estudio, las reducciones fonéticas de adverbios y preposiciones son las variantes regionales predominantes en el corpus $(38,76 \%)$.

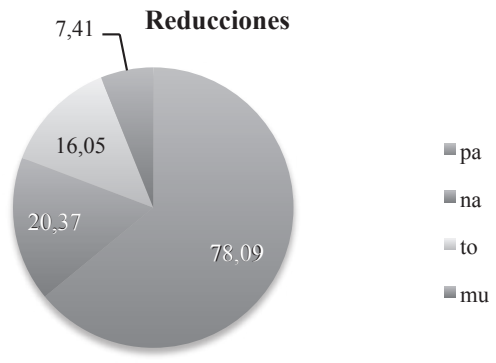

Gráfico 11. Resultados sobre reducciones fonéticas.

16 La zona seseante de Andalucía está compuesta por el área sur de Huelva, el norte de la provincia de Sevilla y algunas áreas de la ciudad de Sevilla; aparece también en el sur de la provincia de Córdoba, incluida la capital, en la parte norte de Málaga, en Jaén y el oeste de la provincia de Granada. El ceceo, en cambio, aparece sobre todo en la franja meridional de Andalucía; se encuentra, igualmente, en el sur de Huelva, la mayor parte de la provincia de Sevilla, la totalidad de la provincia de Cádiz y Málaga, el sur de Granada y en la zona costera occidental de Almería. Las zonas distinguidoras componen, únicamente, un tercio de la comunidad autónoma (Jiménez Fernández, 1999: 32). 
Como se ha afirmado anteriormente, aunque estas variantes forman parte del español coloquial, su extensión es más frecuente en las hablas meridionales, entre las que destacan el extremeño y el andaluz. Este tipo de (orto)grafía fonética incluye, primero, la reducción de la preposición "para", que parece ser la más habitual $(78,09 \%)$ en el corpus analizado; se han encontrado, igualmente, reducciones en adverbios como na 'nada' (20,37\%), to 'todo' $(16,05 \%) \mathrm{y}$, aunque con una menor frecuencia, se han localizado ejemplos de $m u$ para representar el adverbio 'muy' (7,41\%). Además, en los mensajes obtenidos de la red social se han podido advertir algunas reducciones que influyen en transformaciones por fonética sintáctica (para él>pal, todo el $>$ tol) e, incluso, se han encontrado formas como namá 'nada más' que influyen, además, en la representación fonética de la elisión de la -/s/ en posición final.

b. Pérdida de la $-/ d /-$

La ausencia de la -/d/- en posición intervocálica es, junto a las reducciones de adverbios y preposiciones, la representación (orto)regional más común en los mensajes obtenidos de la red social Twitter. En este caso, estudiaremos los contextos en los que desaparece la dental.

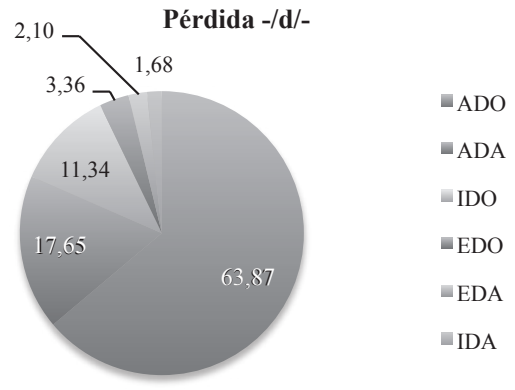

Gráfico 12. Resultados sobre la pérdida de la -/d/-.

Como se puede advertir en el gráfico 12, los resultados se observan, sobre todo, en el sufijo - ado (63,87\%) aunque, igualmente, es relevante la pérdida de la -/d/- en las terminaciones $-a d a$ e -ido. Este hecho, que forma parte, de nuevo, de la conversación coloquial, refleja cómo los hablantes incluyen la oralidad en la escritura de la red social. Además, aunque la ausencia de la dental en posición intervocálica es general sobre todo en los sufijos - $a d o,-a d a$, en el análisis del corpus se han localizado otros casos de pérdida de la -/d/- intervocálica (no tan frecuentes en otras áreas lingüísticas peninsulares) en ejemplos como amargaito 'amargadito', ajustaito 'ajustadito' o destrozaita 'destrozadita', que reflejan la extensión de la pérdida de la -/d/- en los sufijos - ado, -ada en los que se incluye un sufijo diminutivo -ito e -ita respectivamente.

\section{c. Pérdida del consonantismo final}

En cuanto a la representación gráfica de la ausencia de sonidos consonánticos en posición final, en el gráfico 10 se puede comprobar que, en el corpus utilizado, el fenómeno afecta a varios sonidos. 


\section{Pérdida consonantismo final}

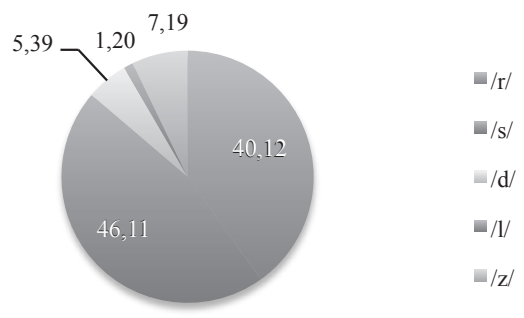

Gráfico 13. Resultados sobre la pérdida de consonantes finales.

La ausencia de grafías para representar la pérdida de consonantes en posición final aparece, fundamentalmente, en los sonidos líquidos -/r/ y -/l/ y dentales -/d/ y -/ / así como en la fricativa -/s/. No obstante, la pérdida no afecta a todos los sonidos de la misma forma; como se puede observar en el gráfico 13, el mayor porcentaje de elisión se presenta en la fricativa -/s/ $(46,11 \%)$ y en la líquida $-/ \mathrm{r} /(40,12 \%)$ y otros casos como la pérdida de la grafía $z$ para representar la interdental o la $d$ para la dental son más aislados $(7,19 \%$ y $5,39 \%$ respectivamente).

Como se puede advertir, por tanto, la inestabilidad de estos sonidos en la lengua oral se reproducen, igualmente, en la escritura en la red. No obstante, es cierto que, ante la ausencia de una grafía que represente el sonido, los usuarios emplean, en ocasiones, recursos gráficos como la reduplicación de vocales (aprendee, doloo, carnavalee, felisidá) o la acentuación de la última vocal de la palabra como ocurre en los casos ejemplos de porfavó, criticá o mujé para la -/r/ en posición final.

\subsection{2. (Orto)grafía regiolectal. Rasgos morfológicos}

Aunque como se advirtió al inicio de esta sección los rasgos morfológicos encontrados fueron escasos, únicamente presentes en el 15,62\% del total de variantes recopiladas, a continuación nos centraremos en el estudio de la presencia de los sufijos diminutivos en la red social para comprobar, en este caso, la frecuencia de uso de este tipo de formas gramaticales (propias de la oralidad) y, además, su extensión geográfica.

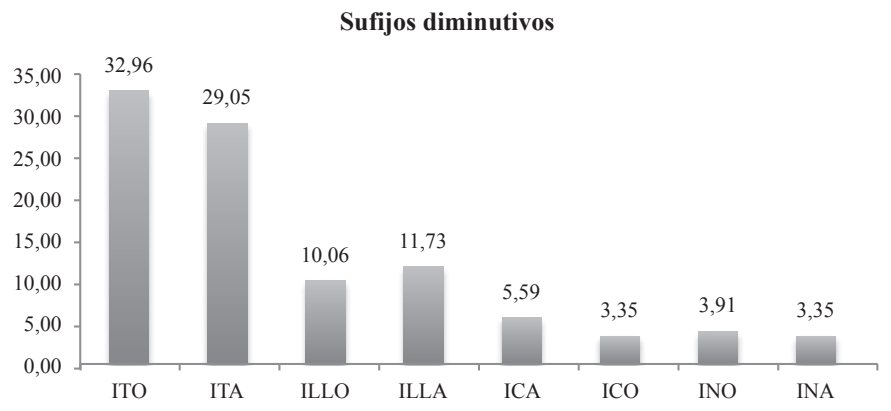

Gráfico 14. Frecuencia de uso de los sufijos diminutivos 
Como se observa en el gráfico 14, los sufijos más habituales en la red social fueron -ito (32,96\%), -ita $(29,05 \%)$ que, además, estuvieron presentes en los comentarios tanto de los extremeños como de los andaluces. En cambio, si observamos los sufijos siguientes, podemos establecer una distribución geográfica del uso de este tipo de formas que remiten, además, a zonas concretas de realización. Por una parte, los sufijos -illo e -illa fueron utilizados en las dos provincias pero, sobre todo, en la región andaluza; en cambio, si analizamos los sufijos-ica $(5,59 \%)$, -ico $(3,35 \%)$, -ino $(3,91 \%)$, -ina $(3,35 \%)$, aunque no son tan habituales su extensión geográfica sí es particular. Frente a -illo, -illa, sufijos que comprobamos que fueron utilizados en ambas regiones, los segundos, -ico, -ica, parecen ser seleccionados, únicamente, por los usuarios de la zona oriental andaluza (en los puntos geográficos de Granada, Almería, Jaén y Baeza) y las formas -ino, -ina fueron elegidas únicamente por hablantes extremeños (aunque encontramos un caso en Andalucía).

Gracias al uso de los sufijos, por tanto, podemos corroborar que en sus mensajes los usuarios de Twitter sí muestran rasgos de oralidad y que, además, este tipo de realizaciones pueden ayudar a establecer variaciones geográficas que determinan la estructura lingüística de ambas regiones.

\subsection{3. (Orto)grafía regiolectal. Léxico y expresiones}

Uno de los testimonios lingüísticos directos del uso del dialecto visual en las redes sociales es el empleo de variantes léxicas y expresiones propias de la región de procedencia de los individuos. En este caso, hay que tener en cuenta que, cuando un usuario de Twitter utiliza una palabra o una expresión regional lo hace, fundamentalmente, con otros usuarios de la misma región porque son estos los que conocen el significado de lo que quiere expresar y, evidentemente, porque la mayor parte de sus redes sociales se encuentran en esa zona geográfica.

Por una parte, y en cuanto al léxico recopilado, podemos destacar ciertas voces propias de las áreas meridionales que, fundamentalmente, han sido consideradas andalucismos pero que, de igual forma, pueden aparecer en el habla de Extremadura. Veamos algunos ejemplos:

(1) no creo killo q lo impidaa jajaja, seria una malaje mu grande... Pero si llueve me Meto debajo un chozajo y to solucionao

(2) \#NoSoportoEsasPersonasQue están todo el día en casa con los \#tacones \#comprateunasbabuchas malajeee

(3) si si eso es verdad son un poquito cansinos jajaja

(4) Venir jarto cerveza, y echarte una siesta de tres horas. \#placercruzcampo

(5) Un poco jartito de ver en todos los canales a cristiano trishte o enfandado. ¿Es que no hay futbol este fin demana?

(6) Toca poner rumbo a Arroyo. Este finde va a ser integro para el rey de mi casa, para mi canijo

(7) Por fin en casa picha QUE DIA MAS LARGO Y QUE PECHA ANDAR

(8)@usuario ${ }^{17}$ pecha de reir examen a las

Como se puede observar en los tuits aportados, hemos encontrado algunas variantes léxi-

17 En los casos en los que se hace referencia a un usuario, en esta investigación hemos preferido guardar su anonimato. 
cas como malaje (ejemplos 1 y 2) que se define como: "dicho de una persona: desagradable, que tiene mala sombra" (RAE, 2001). Según el diccionario académico se trata de un andalucismo que, concretamente, en nuestro corpus, apareció en las áreas de Sevilla y Cádiz.

Otra de las voces encontradas, cansino, vuelve a ser definido como un andalucismo que se refiere a una persona, "cansado, pesado"; en nuestro corpus, en cambio, esta voz se recogió en Almendralejo por lo que puede ser tratado, igualmente, como extremeñismo léxico.

En el ejemplo 4 se puede advertir la palabra jarto, uso que se refiere, en este caso, a una variante fonética de 'harto'; en cambio, esta palabra tiene otra variante semántica en las hablas meridionales que se refiere a "saciado, lleno" pero referido no solo a la comida sino al cansancio, tal como se puede comprobar en el ejemplo 5. Como sinónimo de jarto, podemos encontrar pecha, propuesta en los ejemplos 7 y 8 . En estos casos, y de nuevo como andalucismo, el significado de esta forma se refiere a "panzada, hartazgo" (RAE, 2001); en nuestro corpus, hemos recopilado esta variante en Sevilla y Málaga pero, también, en el área de Cáceres.

Por último, es necesario destacar el uso de canijo para referirse, simplemente, a un apodo o sobrenombre referido a persona; aunque en el caso del diccionario académico esta voz responde a una persona "débil, enfermiza", en las áreas meridionales suele utilizarse como forma afectuosa para denominar a una persona conocida.

Por otra parte, y en cuanto a las expresiones que se han recopilado, podemos resaltar varias de ellas:

(9) que miedo me da esta casa, ofú

(10) ofu que frio

(11) con todos mis respetos tu afición al futbol deja mucho que desear...; ahora anticule... Oju

(12) so guapo

(13) Lo dicho, J.C., telaaaa de mejora en el tema de las bebidas

(14) no entiendo como el Sevilla fc esta en el puesto que esta porque son malos jugando al futbol tela

(15) ala a comer patatas despues de un duro día jajaja

(16) ea tarde de futbol, ... hacer deporte porlo menos se despeja unooo

(17) miarmaa too ere increiblemente increibee

(18) novee que frio hace aquiii

Primeramente hay que hacer referencia a las expresiones ofú, ojú, utilizadas en los ejemplos 9, 10 y 11. Con estas variantes, los usuarios representan situaciones de miedo (ejemplo 9), cansancio (10) e incluso desesperación (11). Otras como la estructura so+adjetivo, que en nuestro corpus apareció representada como so guapo, es un recurso muy frecuente seleccionado, normalmente, junto a adjetivos valorativos. Igualmente, para intensificar un enunciado se utiliza la voz tela $(13,14)$ que, aunque usada como sustantivo, realmente se refiere a un adjetivo como 'mucho'.

El empleo de jala! (15) como variante de la interjección 'venga' es una expresión de origen árabe que ha sido adaptada al español aunque impera en las hablas meridionales; de igual forma, ea (16), como sinónimo de 'bueno', se usa para expresar aprobación o consentimiento de lo enunciado. Otros usos como miarma (17), en este caso con una neutralización de líquidas en la lengua oral y representada, igualmente, con una (orto)grafía fonética en la escritura virtual, es muy frecuente en andaluz; también lo es nove (18), que se refiere a la expresión 'no veas'. 
Finalmente, para completar este apartado, haremos referencia a algunos tratamientos que se han encontrado en el corpus analizado. Como se pudo comprobar en los resultados del cuestionario realizado sobre la conciencia lingüística y el uso de las variantes regionales en las redes sociales, la mayor parte de los informantes no reconocieron usar tratamientos como illo, acho (Gráfico 5). No obstante, en nuestro corpus sí hemos recopilado algunos tratamientos propios de las áreas meridionales, tal como se puede comprobar a continuación:

\section{Tratamientos}

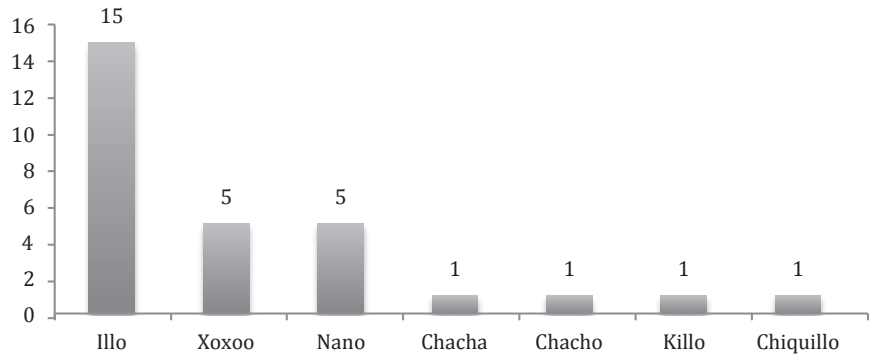

Gráfico 15. Frecuencia de aparición de tratamientos.

En el gráfico 15 se puede advertir las frecuencias absolutas de las formas de tratamiento que recogidas en los mensajes seleccionados ${ }^{18}$. Los datos revelan que el uso de illo y sus variantes (chiquillo, killo) son las más comunes (teniendo en cuenta que la mayor parte de los datos obtenidos proceden de Andalucía, era un resultado esperable). Se observan, además, formas como chacho y chacha, en este caso, localizados en Extremadura pero con una presencia muy escasa. Estos datos revelan que, aunque es cierto que los tratamientos están presentes en nuestro corpus, estas representaciones de las variedades regionales no son tan comunes como, por ejemplo, los rasgos fonéticos lo que corrobora, por tanto, nuestras primeras hipótesis de investigación.

\section{Conclusiones}

Como se ha podido comprobar a lo largo de este trabajo, la oralidad sigue aún hoy muy presente en la red, concretamente, en la escritura de los mensajes de las redes sociales. Según nuestros resultados, la (orto)grafía regional, testimonio directo de este tipo de representación gráfica de la oralidad en la escritura, sigue latente en estas nuevas plataformas de comunicación pero su extensión en Twitter es menor que en otras redes como, por ejemplo, Facebook.

En el corpus utilizado para este estudio se han encontrado este tipo de representaciones gráficas, sobre todo fonéticas; destacan rasgos como la pérdida de la -/d/- en posición intervocálica y del consonantismo en posición final (sobre todo de líquidas $r, l$ y de la fricativa $s$ ), las reducciones de preposiciones y adverbios e, incluso, la presentación de fenómenos meridionales como el seseo o el ceceo, con un predominio evidente del primero; se han

18 Se han utilizado las frecuencias absolutas de los resultados para poder representar la escasa presencia de este tipo de tratamientos en las redes sociales. 
recopilado, igualmente, usos de tratamientos como illo, killo, meridionalismos como canijo, malaje y expresiones como ojú, ofú en los comentarios.

No obstante, y tal como se advirtió al comienzo de esta investigación, el carácter asincrónico de esta red social y el tipo de interacciones que hacen los usuarios al escribir sus tuits influyen notablemente en el uso de un lenguaje mucho más reflexivo que en otras plataformas de comunicación. Estas afirmaciones ya se pudieron comprobar en las encuestas realizadas a los usuarios de redes sociales que afirmaban usar más variantes regionales en Facebook que en Twitter, por ejemplo.

Nuestros resultados avalan que, aunque la escritura oralizada está presente en esta red social, representada en este caso por el uso de variedades regionales, en Twitter existe una menor tendencia a emplear estas variantes que en otras plataformas. En este continuum entre la oralidad y la escritura en el que se incluye la comunicación de esta red social, los individuos suelen tener una mayor conciencia de la distancia comunicativa en el momento de escribir sus mensajes, que son públicos y que, además, pueden ser encontrados mediante el uso de etiquetas; en cambio, existirá una mayor inmediatez comunicativa en las conversaciones con amigos y familiares. Así, aceptando que en Twitter pueden ocurrir ambas situaciones, es lógico que únicamente se haya obtenido menos del $10 \%$ de casos de (orto) grafía regional en la red social pues, en numerosas ocasiones, prima el envío de mensajes públicos frente a las conversaciones entre usuarios conocidos.

Finalmente, y teniendo en cuenta estas consideraciones, sería deseable contrastar estos resultados con una investigación sobre el uso de las variedades regionales en Facebook que permitiera corroborar estas hipótesis o dilucidar nuevos resultados de investigación; este será, sin duda, un campo de estudio probable en próximas investigaciones.

\section{Referencias bibliográficas}

Androutsopoulos, J.K. (2000): "Non-standard spellings in media texts: The case of German fanzines", Journal of Sociolinguistics, 4, págs. 514-533.

Ariza, M. (1987): "La conciencia lingüísitca y el problema del habla de Extremadura". En Viudas Camarasa, A., Salvador Plans, A. y Ariza Viguera, M. El habla en Extremadura, Mérida, Editoria Regional, págs. 21-37.

Blanco, M. J. (2002): “El chat: la conversación escrita”, ELUA, 16, págs. 43-87.

Briz, A. (2010): "El registro como centro de la variedad situacional. Esbozo de la propuesta del grupo Val. Es. Co. Sobre las variedades diafásicas”. En Fonte, I. y L. Rodríguez Alfano (eds.). Perspectivas dialógicas en estudios del lenguaje. México, Universidad Autónoma de Nuevo León, págs. 21-56.

Bustos Tovar, J.J. (1995): "De la oralidad a la escritura”. En Cortés, L. (ed.), El español coloquial. Actas del I Simposio sobre Análisis del Discurso Oral. Almería, Universidad de Almería, págs. 11-28.

Carreras Riudavets, F; Santana Herrera, J.C.; Hernández Figueroa, Z.; Rodríguez Rodríguez, G. (2011): Parametrizador morfológico de textos -ParamText TIP. http://tip.dis.ulpgc.es

Cassany, D. (2011): En-línea. Leer y escribir en la red. Barcelona, Anagrama.

Castañeda, L. y Gutiérrez, I. (2010): "Redes sociales y otros tejidos online para conectar personas". En Castañeda, L. (coord.), Aprendizaje con Redes Sociales. Tejidos educativos en los nuevos entornos. Sevilla, MAD Eduforma. 
Cicognani, A. (1998): “On the linguistic nature of cyberspace and virtual communities", Virtual Reality, 3, págs. 16-24.

Fernández de Molina, E. (2015), "La interacción lingüística en las redes sociales. Aspectos ortográficos", en Montoro del Arco, E., Estudios sobre ortografía del español, Lugo, Axac, págs. 39-51.

Galindo, M. (2014): “Twitter y la Lingüísticia: apuntes para una comunicación 2.0”, en Cuadros, R., Villatoro, J. (eds.). Twitter en la enseñanza y aprendizaje de ELE, Digitalingua, Málaga, págs. 25-46.

Gómez Torrego, L. (2006): "La gramática en internet", Lengua y escritura en internet: tres décadas de "red-acción”. http://congresosdelalengua.es/valladolid/ponencias/nuevas_fronteras_del_espanol/4_lengua_y_escritura/default.htm (1-02-2015).

Jiménez Fernández, R. (1999): El andaluz. Madrid, Arco Libros.

Koch, P. y Oesterreicher, W. (1985): "Sprache der Nãhe-Sprache der Distanz. M ndlichkeit und Schriftlichkeit im Spannungsfeld von Sprachtheorie und Sprachgeschichte", Romanistisches Jahrbuch, 36, págs. 15-43.

Lara, T. (2012): “Twitter y sus funciones comunicativas”, BlogTíscar.com. http://tiscar. com/2012/03/11/twitter-y-sus-funciones-comunicativas $>(1-02-2015)$.

López Quero, S. (2004): "Foros de debate vs. Otros discursos electrónicos”, “Twitter y la Lingüísticia: apuntes para una comunicación 2.0”. Español actual: Revista de español Vivo, 82, págs. 53-76.

López Quero, S. (2013): "La conversación escrita en internet: caracterización pragmalingüística”, Sintagma 25, págs. 77-92.

Mancera Rueda, A. y Pano Alamán, A. (2013): El español coloquial en las redes sociales. Madrid, Arco Libros.

Mancera Rueda, A. y Pano Alamán, A. (2014): "Las redes sociales como corpus de estudio para el análisis del discurso mediado por ordenador”, Humanidades digitales: desafios, logros y perspectivas de futuro. Janus, Anexo 1, págs. 305-315.

Merchant, G. (2001): "Teenagers in Cyberspace: an investigation of language use and language change in internet chatrooms", Journal of Research in Reading, 24 (3), págs. 293-306.

Martínez de Sousa, J. (2004): Ortografía y ortotipografía del español actual. Gijón, Trea.

Stein, D. (2006): "E-mail, Internet, chatroom talk: Pragmatics". En Keith Brown (ed.) Encyclopedia of Language and Linguistics (2nd edition), Amsterdam, Elsevier, págs. 116-124.

Tascón, M. (2012): Escribir en internet. Guía para los nuevos medios y las redes sociales. Barcelona, Galaxia Gutenberg.

Yus, F. (2001): Ciberpragmática. Barcelona, Ariel.

Yus, F. (2011): Ciberpragmática 2.0: Nuevos usos del lenguaje en internet. Barcelona, Ariel 